
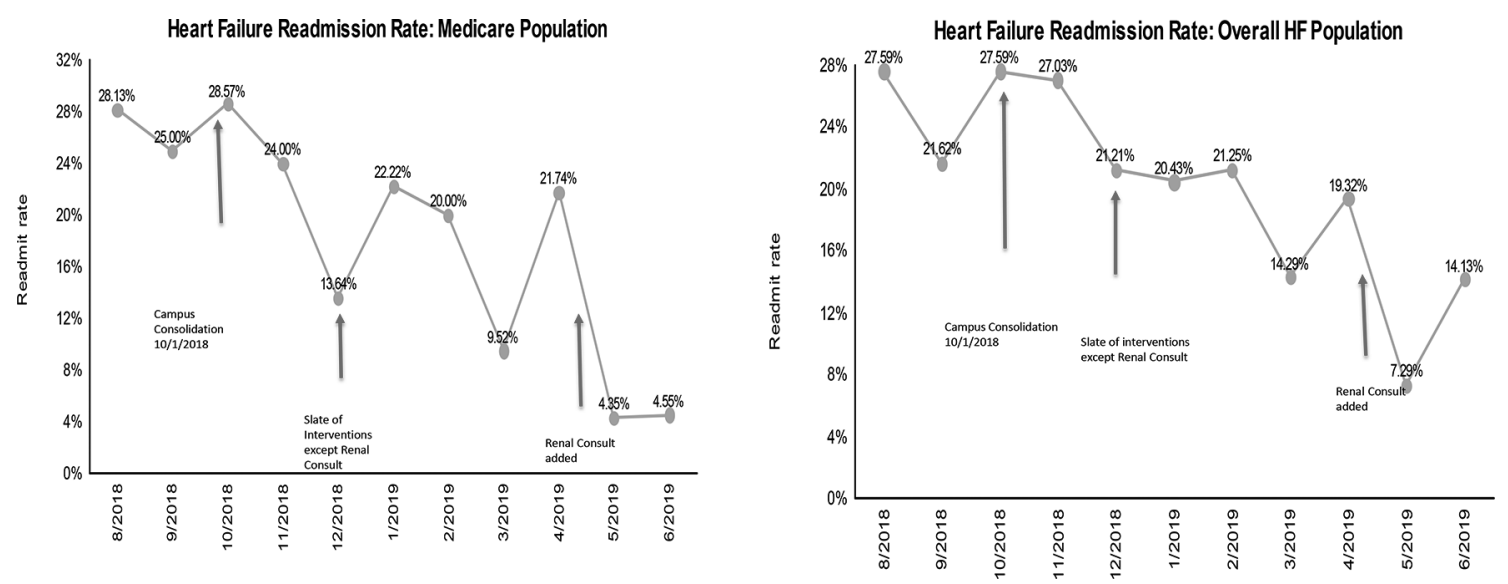

Abstract 29 Figure 1

\% HF Consult on Non Cardiology

Teams

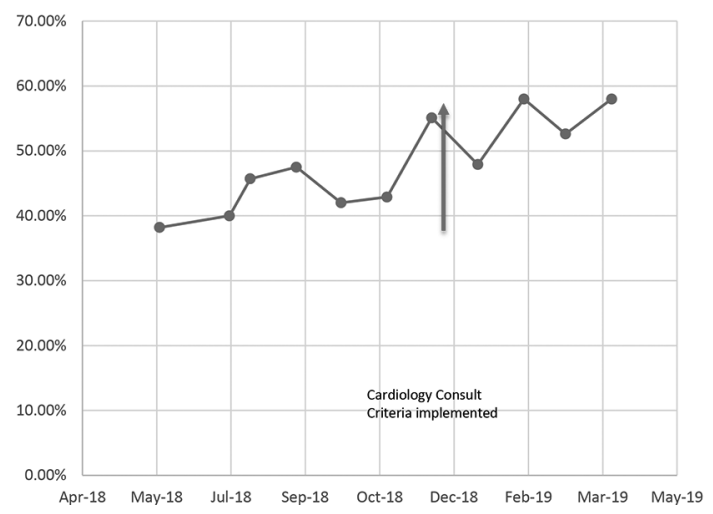

Abstract 29 Figure 2

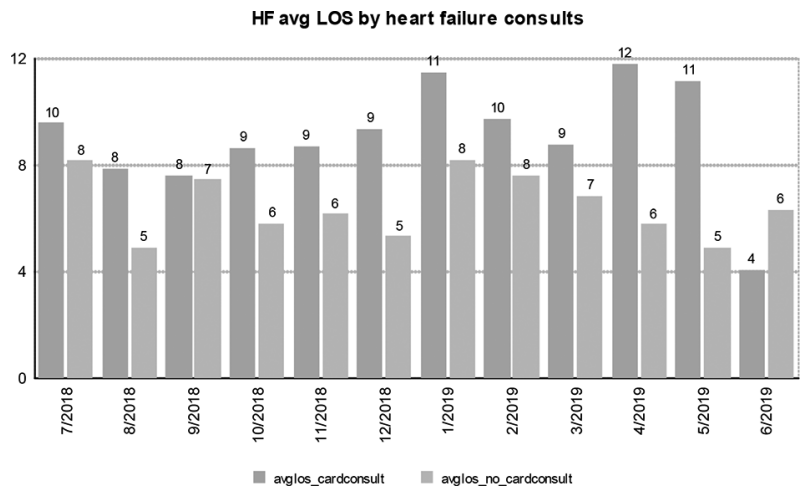

Abstract 29 Figure 3

Results In the six month prior to QI initiative, BMC's Medicare 30 day HF readmission rate averaged $26.85 \%$ which decreased to $15.2 \%(12 / 1 / 18-6 / 30 / 19)$ as the slate of interventions fully activated. Total HF readmissions showed similar decrease from $23.9 \%$ to $17.2 \%$.

Conclusions BMC demonstrates that meaningful Heart Failure readmission reductions can be achieved Safety Net Hospitals by tapping into existing institutional resources in new ways. Planning permanent accountable team, automated dashboards and robust feedback loops insure reductions seen are sustained.

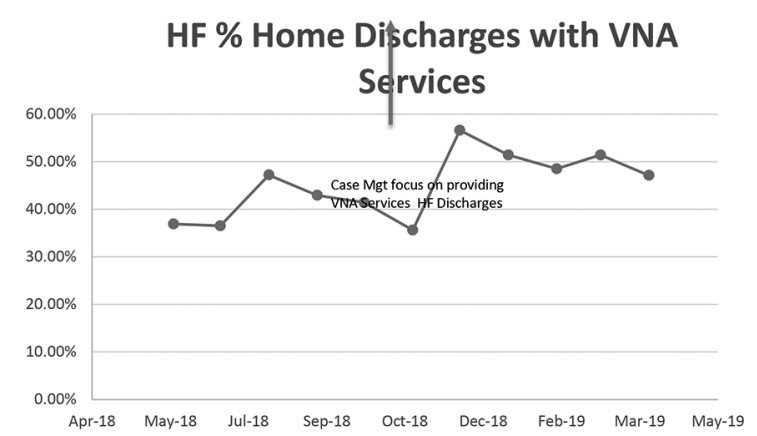

\section{REDUCING IATROGENIC LOW BLOOD GLUCOSE EPISODES DURING HOSPITAL MANAGEMENT OF PEDIATRIC DIABETIC KETOACIDOSIS}

${ }^{1}$ Shannon Baumer-Mouradian, ${ }^{2}$ Ashley Servi, ${ }^{2}$ Pam Gage, ${ }^{2}$ Sandy Huitink, ${ }^{2}$ Rebecca Sprague, ${ }^{2}$ Jeremy Ligget, ${ }^{1}$ Peter Wolfgram. ${ }^{1}$ Medical College of Wisconsin, USA; ${ }^{2}$ Children's Hospital of Wisconsin, USA

\subsection{6/bmjoq-2019-ihi.30}

Background IV insulin therapy, the treatment for pediatric diabetic ketoacidosis (DKA), may lead to iatrogenic low blood glucose episodes (BG $<100 \mathrm{mg} / \mathrm{dL}$ ). At our institution, $53 \%$ of DKA patients on IV insulin experienced BG $<100 \mathrm{mg} / \mathrm{dL}$ episode resulting in premature discontinuation of insulin and delays in therapy.

Objectives We aimed to decrease the percent of DKA patients experiencing BG $<100 \mathrm{mg} / \mathrm{dL}$ episodes while on IV insulin from $53 \%$ to $43 \%$ over 24 months.

Methods Multiple interventions linked to key drivers were tested using plan-do-study-act cycles including a standardized DKA guideline incorporating the two-bag fluid system, order panels, frequent blood glucose monitoring, and provider and nursing education. The primary outcome was the percent of DKA patients experiencing a BG $<100 \mathrm{mg} / \mathrm{dL}$ episode while on IV insulin. Order panel utilization rate was the process measure. Balancing measures included length of stay, time to correction of acidosis (time to HCO 3>17), and admission 


\section{Percent of DKA Patients with an Episode of Blood Glucose $<100$ P Chart}

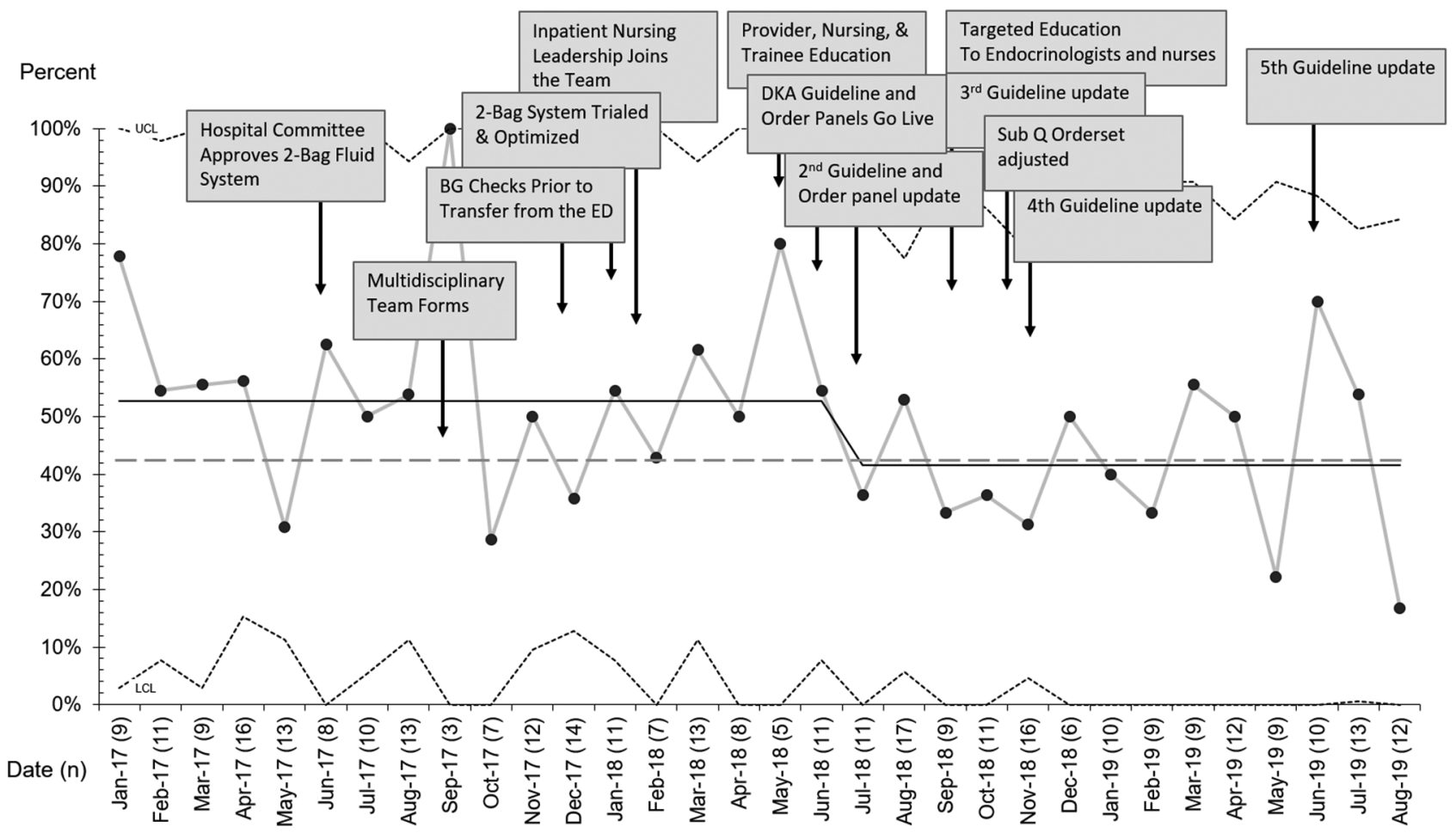

Abstract 30 Figure 1 Percent of DKA patients with an episode of blood glucose $<100 \mathrm{P}$ chart

rates. All outcomes were analyzed on statistical process control charts.

Results From January 2017 through August 2019, 334 patients had confirmed DKA (bicarbonate level <15) and received IV insulin therapy in the ED or on the acute care unit. Following our interventions, the number of patients experiencing a low blood glucose level improved from $53 \%$ to $42 \%$ (figure 1 ). Order panel utilization rate remained at $96 \%$. There was no change in our balancing measures.

Conclusions Use of QI methodology and standardized DKA management resulted in a meaningful reduction in the number of patients with a $\mathrm{BG}<100 \mathrm{mg} / \mathrm{dL}$ episode while on IV insulin.

\section{REDUCING VARIABILITY IN DISCHARGE COMMUNICATION REDUCES ADMINISTRATIVE BURDEN}

Stephanie Lumpkin, lan Kratzke, Clark Howell, Nicole Chaumont. University of North Carolina, USA

\subsection{6/bmjoq-2019-ihi.31}

Background Streamlined discharge communication in transitions of care improvement models improve patient satisfaction, improve patient self-efficacy, and reduce readmissions. Yet, significant variability and quality exists in the After Visit Summary (AVS) which is used to guide the post-discharge needs of the patient.

Objectives We sought assess the feasibility, fidelity, acceptability, and effectiveness of a standardized, patient-centered AVS.

Methods Our multiprofessional and multidisciplinary stakeholder team was led by two physicians and had significant institutional support. We employed a tailored, hybrid implementation strategy, blending components from Lean methodology, the Institute for Healthcare Model for Improvement, and the Consolidated Framework for Implementation Research. We assessed the feasibility (e.g. quality of AVS, usability of template), fidelity (e.g. template utilization rates), acceptability (e.g. direct stakeholder feedback, paging-system audit, patient call logs), and effectiveness (e.g. 30-day readmission rates and patient-centeredness) of our intervention (e.g. standardized, patient-centered AVS).

Results Our AVS template was written at a third grade reading level but retained all clinically-relevant information. Providers and patients approved of the template with $66 \%$ mean utilization (range $41 \%$ to $80 \%$ ). After our intervention, we observed decreased 30-day readmissions $(19.7 \%$ to $16.0 \%)$. Notably, after the introduction of AVS template, pages sent decreased by 3.67 pages weekly $(95 \% \mathrm{CI}=[-15.7,14.0])$, but this estimate was imprecise.

Conclusions Reducing variability and improving the quality of discharge communication, not only improves patient outcomes, but may also improve workflow for providers. These improved team dynamics are important to assess when considering an intervention that is operationalized by time and resource deprived employees.

\section{THE 4 MS STRATEGY TO FALL PREVENTION}

William Barnett, Michael Dodrill, Carson Oostra, Mujahed Alkhathlan, Zeid Nesheiwat, Christian Nehme, Ammar Kayyali. University of Toledo, USA

\subsection{6/bmjoq-2019-ihi.32}

Background Despite the fact that many organizations have implemented fall risk scales to identify patients at risk, fall 